\title{
LAZER KAPLAMA PROSESİ ve PROSES PARAMETRELERİ: DERLEME ÇALIŞMASI
}

Çiğdem DINDAR*

Meryem ALTAY

Hakan AYDIN

Öz: Günümüz lider üretim teknolojilerinden biri olan lazer kaplama prototip, onarım ve imalat uygulamalarında kullanılmakta olup havacılık, uzay, otomotiv, savunma sanayi, tıp vb. alanlarda geniş uygulama alanına sahiptir. Üretim yöntemi, lazer ışını kullanılarak toz malzemenin ergimesi ve temel malzeme ile nüfuziyetine dayanmaktadır. Lazer kaplama prosesinde, malzemenin nihai kalite özellikleri işlem parametrelerinden doğrudan etkilenmektedir. Bu parametrelerin önemi, malzeme makro ve mikro yapısının incelenmesi üzerine yapılan çalışmalarla ortaya konulmaktadır. Bu derleme çalışmada, lazer kaplama prosesi detaylı olarak incelenerek lazer gücü, ilerleme hızı, toz besleme hızı ve koruyucu gaz proses parametrelerinin kaynak bölgesine etkileri irdelenmiş olup literatürde yer alan sonuçlar derlenmiştir.

Anahtar Kelimeler: Lazer kaplama, Eklemeli imalat, Proses parametreleri, Malzeme onarımı.

\section{Laser Cladding Process and Process Parameters: A Review}

\begin{abstract}
Laser cladding method, which is the one of leading manufacturing technologies of today, is used in various applications such as prototyping, product repair and material coating. It has a wide application areas: aviation, space, automotive, defense industry, medicine, etc. The production method is based on the melting of the powder material using a laser beam and its penetration with the substrate. In the laser cladding process, the final quality properties of the material are directly affected by the process parameters. The importance of these parameters is revealed by the studies on the macrostructure and microstructure of the material. In this review article, the laser cladding process has researched in detail, the effects of laser power, welding speed, powder feed rate, shielding gas process parameters on the welding area have examined, and the results obtained have given by gathered together.
\end{abstract}

Keywords: Laser cladding, Additive manufacturing, Process parameters, Repair of material.

\section{GíRIş}

Lazer kaplama teknolojisi, gelecek vaat eden etkili bir yüzey modifikasyon tekniğidir (T. Chen ve diğ., 2019). Üretim yöntemi prensibi, toz malzemenin ergiyerek temel malzeme ile doğrudan nüfuziyetine dayanır: metalik toz, malzeme besleme sistemi ile bir nozuldan temel malzemeye aktarılır, aynı anda lazer ışını ile metalik tozların ergimesi sağlanır (Li ve diğ., 2020).

\footnotetext{
* TOFAŞ Ar-Ge Yeni Yalova Yolu Cad. No:574 Bursa/TÜRKIYE

*** Bursa Uludağ Üniversitesi, Mühendislik Fakültesi, Makine Mühendisliği Bölümü 16059 Nilüfer/BURSA

İletişim Yazarı: Çiğdem DİNDAR (cigdem.dindar@tofas.com.tr)
} 
Lazer kaplama prosesinin içerisinde bulunduğu, Amerikan Test ve Malzeme Topluluğu (ASTM) tarafından sinıflandırılan eklemeli imalat yöntemleri Şekil 1'de gösterilmektedir (Singh ve Ramakrishna, 2017). Bu prosesin diğer yöntemlerden temel farkı, malzeme ergitmede lazer ışını kullanmasıdır.

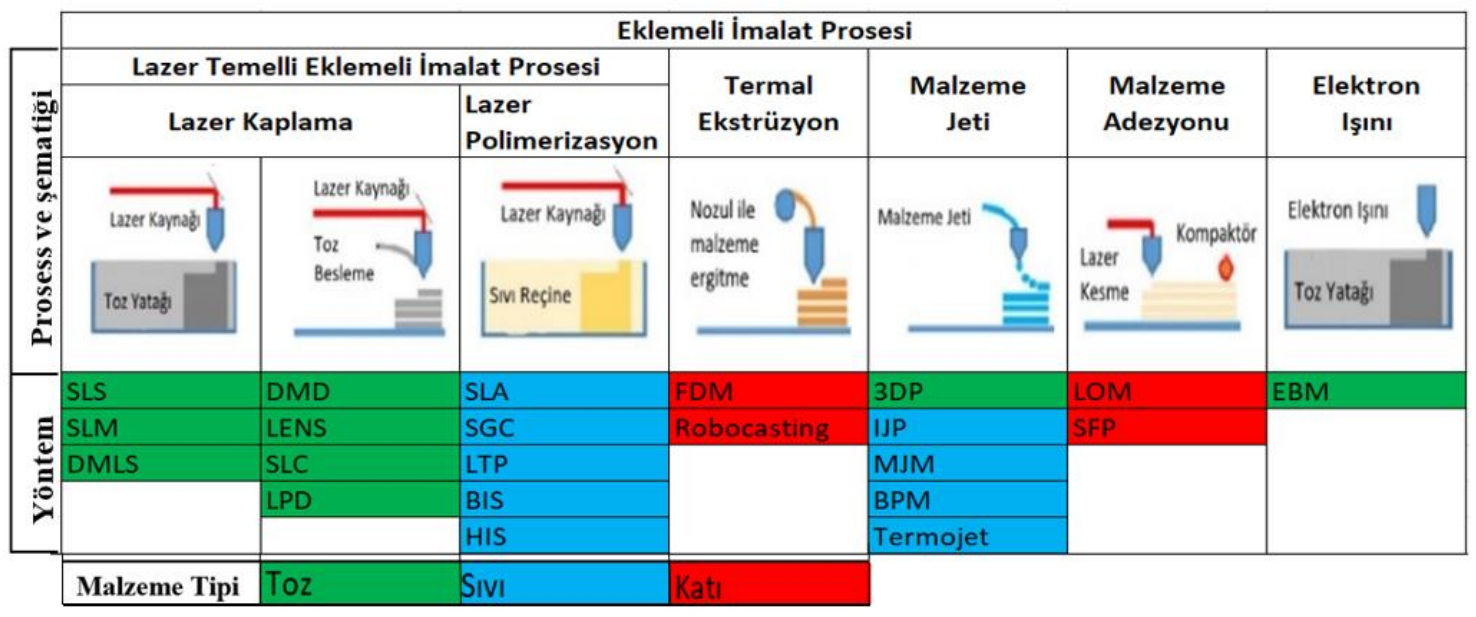

Şekil 1:

Eklemeli imalat teknolojisi lazer kaynak sinıflandırması (Singh ve diğ., 2017)

Son yıllarda lazer kaplama teknolojisi, aşınma direnci (Huang ve di $\breve{g} ., 2020$; Quazi ve di g., 2016), korozyon direnci (Fu ve diğ., 2020; Roy ve diğ., 2020; Wang ve diğ., 2020), oksidasyon direnci (Li-Yan ve diğ., 2020), 1sıl yorulma dayanımı (Liu ve diğ., 2017) ve biyouyumluluk (Zheng ve diğ., 2008) gibi özellikleri elde etmek için uzay, havacılık, enerji, tıp, otomotiv ve makine sektörlerinde kullanılmaktadır (Zhu ve diğ., 2020). Ayrıca uçak iniş takımları ve gövde bileşenleri (Barr ve diğ., 2018), medikal implant (Weng ve diğ., 2014), türbin kanadı (Kaierle ve diğ., 2017), otomotiv çelikleri (Shi ve diğ., 2017) ve kalıp endüstrisinde de (Theron ve diğ., 2020) yaygın kullanımı bulunmaktadır.

Lazer kaplama küçük alanları iyileştirmek için son derece uygundur; belirli yüzey kalitesinde kaplama gereksinimlerini karşılayarak, yüzey kusurlarını onarmak için ideal bir yöntemdir. Ürünlerin hızlı bir şekilde imal edilebilmesi, teknik ve kalite gerekliliklerini karşılayabilmesi gibi yüksek kazanımlara sahiptir (Lourenço ve diğ., 2016). İyileştirme ve onarım uygulamaları birçok komponent için bulunmaktadır: kalıp (Chen ve diğ., 2014; Lu ve

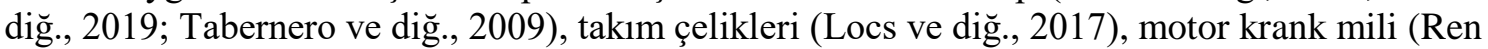
ve diğ., 2013), demiryolu tekerleri (Zhu ve diğ., 2019), dişli çarklar (Liu ve diğ., 2017).

Geleneksel onarım yöntemlerine göre birçok avantaj1 bulunmaktadır: kontrollü 1sı girdisi, yüksek boyutsal doğruluk, esnek üretim yöntemi olması (Riveiro ve diğ., 2014), mekanik ve kimyasal özelliklerin geri kazanılabilmesi nedeniyle tercih edilmektedir (Liu ve diğ., 2017). Bahsedilen avantajlarının yanı sıra; onarım, imalat ve prototip aşamalarında dezavantajları da mevcuttur. Kaplamanın homojen olmaması yüzey kalitesini düşürmekte; gözenek (Zhan ve diğ., 2019), çatlak (Yu ve diğ., 2013), kalıntı gerilme (Chen ve diğ., 2012) gibi olumsuz özellikler oluşturabilmektedir. Ayrıca proses parametrelerinin optimize edilmesi de gerekmekte olan önemli bir husustur.

Lazer teknolojisi kullanılarak yapılan çalışmalarda hafif metaller, çelik ve dökme demirler (Bartkowski ve diğ., 2015), kompozit ve seramik malzemeler (Al-Hamdani ve diğ., 2020; Bu ve diğ., 2020; Chen ve diğ., 2020; Li-Yan ve di ̌̆., 2020; Zhao ve diğ., 2020a) ve pek çok malzemenin yüzey özelliklerini değiştirmek mümkün olmaktadır. 


\section{LAZER KAPLAMA PROSESI}

Lazer kaplama prosesi, yüksek erime noktasına sahip malzemeleri dahi eritebilen güçlü bir lazer kaynağı ve besleme sisteminden (tel ya da toz malzeme) oluşan çok yönlü bir tekniktir (Marin ve dĭg., 2020); lazer dolgu kaynağı ve doğrudan lazer metal biriktirme isimleriyle de bilinmektedir. Düşük 1sı girdisi, yüksek katılaşma oranı, yüksek kaynak nüfuziyeti, fonksiyonel dereceli malzemelerin biriktirilmesi ve homojen yapı elde edilmesi özellikleri nedeniyle tercih edilmektedir (Toyserkani ve di ̌̆., 2017).

Lazer kaplama işleminde, yüksek güçteki lazer ışın kaynağı sayesinde ince bir tabaka (0.05$2 \mathrm{~mm}$ ) oluşturularak kaynak gerçekleştirilir, Şekil 2'de lazer kaplama teknolojisi ve prensibi gösterilmiştir. Lazer başlığından çıkan lazer ışını, nozuldan aktarılan toz malzeme ve koruyucu gaz aynı anda temel malzemenin üzerine gönderilir; lazer 1şını sayesinde toz ve temel malzeme ergiyerek kaynak havuzu oluşturur. Kaplama yapılmak istenilen bölge üzerinde lazer başlığ ilerletilerek işlem sürdürülür. Koruyucu gaz ergiyik havuzundaki malzemeyi oksitlenmeye karş1 korumaktadır ve genellikle Argon gazı kullanılmaktadır (Zhu ve diğ., 2020; Li ve diğ., 2020).

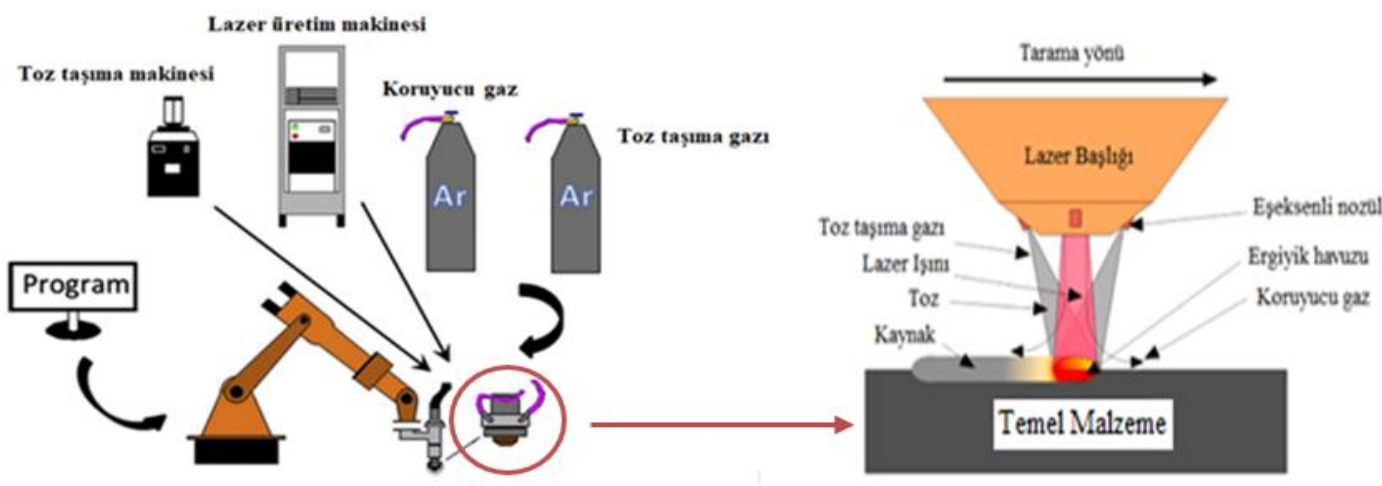

Şekil 2:

Lazer kaplama prosesi (Zhu ve diğ., 2020) ve çalışma prensibi (Li ve diğ., 2020).

Lazer kaplama işleminde üç durum büyük öneme sahiptir: ergiyik havuz, toz akış dinamikleri ve kaplama özellikleri. Kaynak havuzunda gerçekleşen hızlı katılaşma, güçlü sıvı konveksiyonu, yüzey gerilimi kaynaklı marangoni akışı (Jiang ve diğ., 2020), basınç değişimi kaynaklı sıçrama (spatter) etkisi (Prasad ve diğ., 2020) ve difüzyonlar gibi taşınım olayları kaplama tabakasının kompozisyonunu ve mikroyapısını belirler (Zhao ve diğ., 2020b). Ergimiş yüzey katılaştıktan sonra, istenen özelliklere sahip bir katman elde edilmiş olur (Shamsaei ve diğ., 2015); Zhao ve diğ., 2020b). Burada karmaşık olarak gelişen toz akış davranışı, nozül ile etkileşime giren lazer ve temel malzeme, 1s1 transferi ve kaynak havuzundaki olayların anlaşılması oldukça güçtür; dolayısıyla deneysel çalışmaların yanı sıra modelleme çalışmaları da yapılmıştır. Kaynak havuzu, toz akışı ve mikroyapı özelliklerini araştırmak için nümerik model oluşturularak simülasyonlar üzerinde çalışmalar yapılımıştır (Huang ve diğ., 2016; Zhang ve diğ., 2016).

Lazer kaplama prosesinde, kaplama malzemesinin beslenmesi farklı yöntemler kullanılarak gerçekleştirilebilmektedir. Beslenme yollarına bağlı olarak Şekil 3'de görüldüğü gibi dört tipte kategorize edilebilir: önceden yerleştirilmiş toz sistemi (Muvvala ve diğ., 2017), eşeksenli toz sistemi (Devojno ve diğ., 2018), eksen dış1 toz sistemi (Hofman ve diğ., 2011) ve tel besleme sistemi (Xu ve diğ., 2018). Şekil 3(a), önceden yerleştirilmiş toz sistemini göstermektedir; tozlar 
temel malzeme üzerine önceden yerleştirildikten sonra lazer 1şını gönderilerek kaynak işlemi gerçekleştirilir. Şekil 3(b) 'de gösterildiği gibi lazer ışını ve toz besleme sistemi aynı eksenlidir (koaksiyal). Şekil 3(c), toz malzeme kaynak havuzuna lazer ışınının ekseni dışında bir nozul ile aktarılır. Şekil 3(d) tel besleme sisteminde toz malzeme yerine kaplama malzemesi olarak tel malzeme kullanılır. Toz partikülleri boyutları 50 ila $150 \mu \mathrm{m}$ arasında değişir ve tel çapı $1 \mathrm{~mm}$ civarındadır (Tamanna ve diğ., 2019).
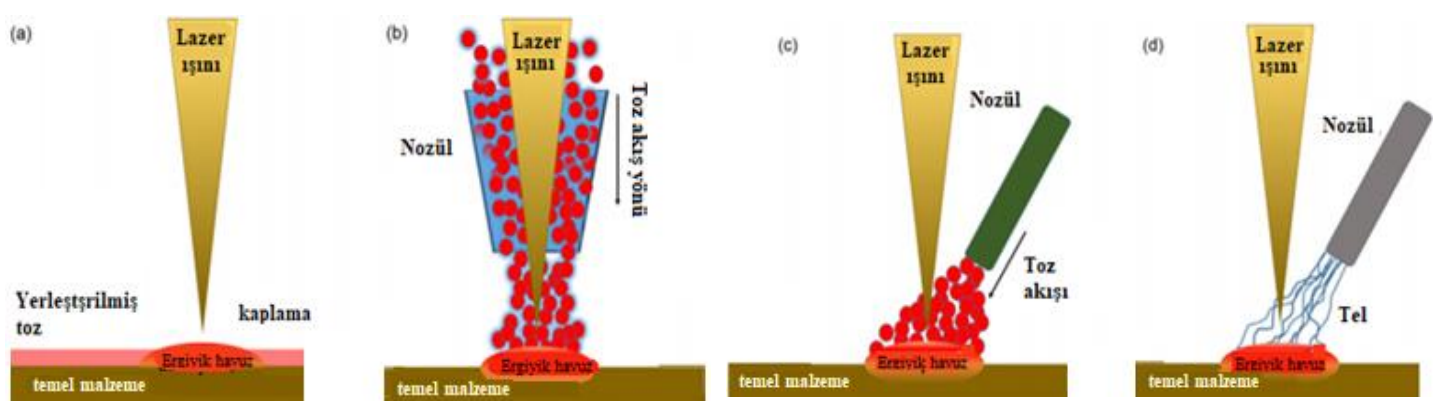

Şekil 3:

Lazer dolgu kaynağı besleme yöntemleri (a) önceden yerleştirilmiş toz sistemi, (b) eşeksenli toz sistemi, (c) eksen dışı toz sistemi, (d) tel besleme sistemi (Tamanna ve diğ., 2019).

Eşeksenli lazer kaplama işlemi, diğer üç besleme yöntemine göre kusursuz besleme sağladığı için öne çıkmaktadır, Şekil 4(a)'da şematik diyagram gösterilmektedir. Bu işlemde, toz besleyici ve lazer sisteminden oluşan eşeksenli nozul Y doğrultusu boyunca tozları taşırken $\mathrm{Z}$ doğrultusunda hareket etmektedir; kaynak işlemi yapılan temel malzeme sabit konumdadır (Ma ve di ̌̆., 2020). Kaplama proseslerinde lazer kaplama bölgesi, 1sı tesiri altındaki bölge (ITAB) ve temel malzeme bölgeleri oluşmaktadır; Chew ve diğ. (2015) yapmış oldukları çalışmada bu bölgeler Şekil 4(b)'de gösterildiği üzere şematik olarak verilmiştir. Şematikte gösterilen her bölgenin özellikleri birbirinden farklıdır ve optik mikroskop incelemelerinde elde edilen görsellerde de bu bölgeler kolaylıkla ayırt edilebilmektedir. Katman sayısının birden fazla olduğu lazer kaplama işleminin yanısıra, her katmanın farklı yönlerde ve farklı geometrilerde olduğu uygulamalar da mevcuttur; dişli çark üzerine farklı geometrilerdeki uygulama Şekil 4(c)'de gösterilmektedir (Yu ve diğ., 2018). 




b)
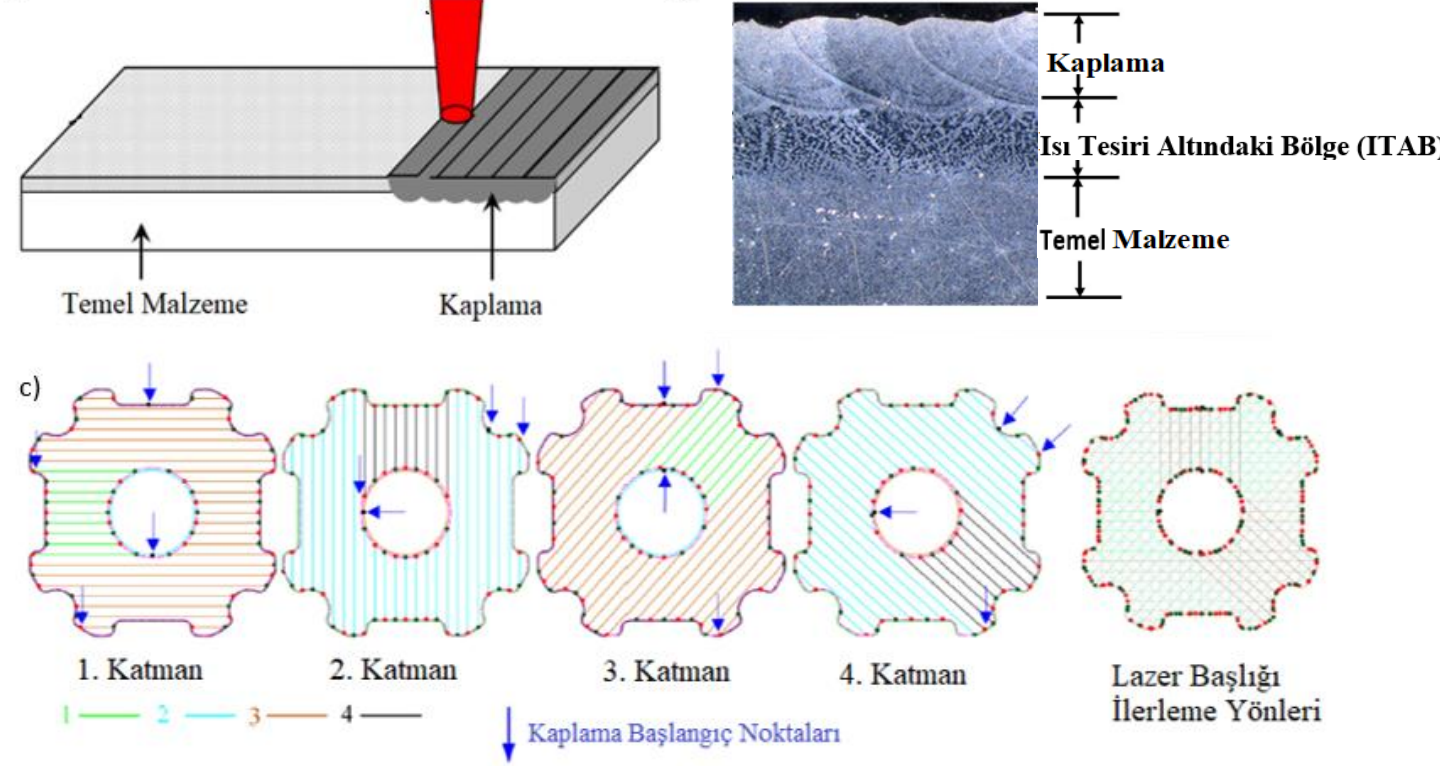

Şekil 4:

(a) Eşeksenli lazer kaplama işleminin şematik diyagramı (Ma ve diğ., 2020),(b) kaplama bölgesi, isı tesiri altındaki bölge ve temel malzeme şematik gösterimi (Chew ve diğ., 2015), (c) katmanların farkl yönlerde ve geometrilerde olduğu, dişli çark lazer kaplama uygulaması (Yu ve di ̌̆., 2018).

\section{LAZER KAPLAMA PROSES PARAMETRELERİ}

Lazer kaplama teknolojisinde, lazer ışınının temel malzemeyi eritip kaynak havuzunun oluşması ve eriyiğin katılaşma sürecinde lazer gücü, ilerleme hızı ve toz besleme hızı parametreleri etkilidir. Değişken proses parametreleri kaplama geometrisini etkilemekte, malzemenin makroyap1, mikroyapı, çekme dayanımı, korozyon direnci ve yorulma dayanımı gibi mekanik özelliklerinde değişikliğe neden olmaktadır (Shamsaei ve diğ., 2015). Lazer kaplama işleminde hem proses parametreleri hem de malzeme karakteristikleri mekanik ve fiziksel özellikler üzerinde önemli etkilere sahiptir (El Cheikh ve diğ., 2012; Hemmati ve diğ., 2011; Shi ve diğ., 2018; Sun ve diğ., 2016).

\subsection{Proses Etkin Parametreleri: Lazer Gücü, İlerleme Hızı, Toz Besleme Hızı}

Lazer gücü kaplama kalitesi üzerinde en önemli parametredir. Şimşek T. ve diğ. (2019) düşük lazer gücü ile yaptıkları deneylerde temel malzeme ile kaplama malzemesi arasında nüfuziyet gerçekleşmemiştir. Kaplama tabakasında yoğun çatlak ve gözenek problemlerinin varlığı tespit edilmiş; nanopartiküllerin yüzeylerde homojen olarak dağılmayıp topaklaşma oluşturduğu gözlemlenmiş̧ir.

İlerleme hızı ve lazer gücü parametreleri oluşturdukları enerji girdisi sayesinde ergiyik havuzunun boyutunu belirleyerek kaplama kalitesi üzerinde etkide bulunmaktadır. Lazer gücünün artmasıyla enerji girdisi artmakta, lazer gücü artışı ile azalmaktadır (Eşitlik 1) (Moskal ve diğ., 2020). İlerleme hızının artışı etkileşim süresince kaynak havuzundaki ergimiş malzeme miktarında azalmaya yol açmaktadır. 


$$
\text { Enerji Girdisi }\left(J / \mathrm{mm}^{2}\right)=\frac{\text { Lazer Gücü }(W)}{\text { İlerleme } \operatorname{Hız\imath }(\mathrm{mm} / \mathrm{s}) * I \operatorname{ssln} \operatorname{Çapı}(\mathrm{mm})}
$$

Toz besleme hızı parametresi ise ısıdan etkilenen bölgenin boyutunu belirlemede hacimsel 1s1 girdisi üzerinde bir etkiye sahiptir. Yüksek toz besleme hızı ergime gerçekleşmeden toz malzemenin uzaklaşarak ergiyik havuzu kesitinin küçülmesine neden olmaktadır. Ergimemiş toz parçacıkları hızlı katılaşarak gazların hapsolmasıyla birlikte gözenekli bir yapı oluşumuna neden olmaktadır (Karşı, A. 2019).

Kaynak yapılabilirlik, sıvı fazın yüzey gerilimi, termal iletkenlik, lazer ışııının yansıma ve soğurulması gibi mazleme özellikleri de lazer kaplama prosesini etkilemektedir (Cavaliere 2021). Ayrıca ilerleme yolunun değişmesi, kaynak havuzu sıcaklığı ve iş parçasının sıcaklık dağılımını etkileyerek mikroyapı ve kalıntı gerilme miktarını değiştirmektedir. Isı girdisinin yüksek olması malzemelerin mekanik özelliklerini olumsuz yönde etkileyerek kaplama tabakasında tane irileşmesine neden olmaktadır ( $\mathrm{Li}$ ve diğ., 2019).

\subsection{Lazer Kaplama Geometrisi}

Yapılan bazı çalışmalara göre lazer gücü, toz debisi, ilerleme hızı, odak mesafesi kaplamanın kalitesi üzerinde büyük etkileri olmuştur. Chen ve diğ., (2019) ve benzer araştırmalarda bulunan Cheikh ve diğ. (2012); lazer gücü, ilerleme hızı ve besleme hızı parametreleri ile kaynak genişliği, kaynak yüksekliği, kaynak alanı ve penetrasyon derinliği arasındaki ilişkileri araştırmışlardır. Çalışmalarında lazer gücü ve besleme hızının artmasının ve ilerleme hızının düşmesinin kaplama kalınlığını artırdığını gözlemlemişlerdir.

Kattire ve diğ. (2015) yapmış oldukları çalışmada, kalıp onarım uygulamaları için bir takım çeliği üzerine bir çelik tozu lazer kaplamasının deneysel karakterizasyonunu araştırmış; lazer parametrelerinin kaplama geometrisi ve kaplama kalitesi üzerindeki etkisini ortaya koymuşlardır. Çalışmalarında lazer gücü ve ilerleme hızı, enerji girdisi ile ilgili önemli parametrelerdir; toz akış hızı, lazer ışınını zayıflatma etkisi nedeniyle önemli olmuştur. Kaplama yüksekliği, lazer kaynağının ilerleme hızındaki artışı ile azalma göstermiştir. Toz besleme hızı düşük olduğunda da kaplama yüksekliği nispeten azdır; temel malzemenin önemli ölçüde erimesi nedeniyle yapışma yüksektir. Toz akış hızı arttıkça, birim uzunluk başına sağlanan artan toz hacmi nedeniyle daha kalın bir kaplamanın oluştuğunu açıklamışlardır (Kattire ve diğ., 2015).

Lazer kaplama işleminde katman sayısı, kaynak genişliği ve kaynak yüksekliğini değiştirdiğinden parçanın hassasiyetini etkilemektedir. Katmanlı olarak biriken malzemenin hassasiyetini arttırmak için Gao J. ve diğ. (2020) parçaların kesit boyutları üzerindeki etki faktörleri hakkında çalışmalarda bulunarak optimum proses parametrelerini araştırmışlardır. Lazer kaplama işlemi sırasında, kaplama alanındaki sıcaklığın kontrol edilmesi de önem arz etmiştir.

\subsection{Proses Parametre Optimizasyonu}

Proses parametrelerinin optimizasyonu ile nitelikli istenen yap1 ve malzemelerin araştırılmasına yönelik birçok çalışma (Chen ve diğ., 2019; Li ve diğ., 2019; Reddy ve diğ., 2018; Shamsaei ve diğ., 2015) yapılmıştır.

Lazer kaplama işleminde, çeşitli deneysel - istatistiksel, analitik ve sayısal modeller oluşturulabilmektedir. $\mathrm{Bu}$ modeller, esas olarak eklemeli imalatın doğrudan enerji biriktirme işlemlerinden olan lazer kaplama ile uygulanabilen kaplama geometrilerini, kaplama özelliklerini, toz akış debisini ve termal profili tahmin etmek için kullanılmaktadır (Zareh ve Urbanic, 2020). Bu modeller için kullanılan yöntem, işlem parametreleri ve elde edilen sonuçlar Tablo1'de verilmiştir (Farahmand ve diğ., 2014; Huang ve diğ., 2016). 
Tablo1. Eklemeli imalat yönteminde lazer kaplama ile mevcut uygulanabilen modellerde, uygulama metodu, proses parametreleri ve ölçülebilecek çıktıların şematik gösterimi (Farahmand ve diğ., 2014; Huang ve diğ., 2016).

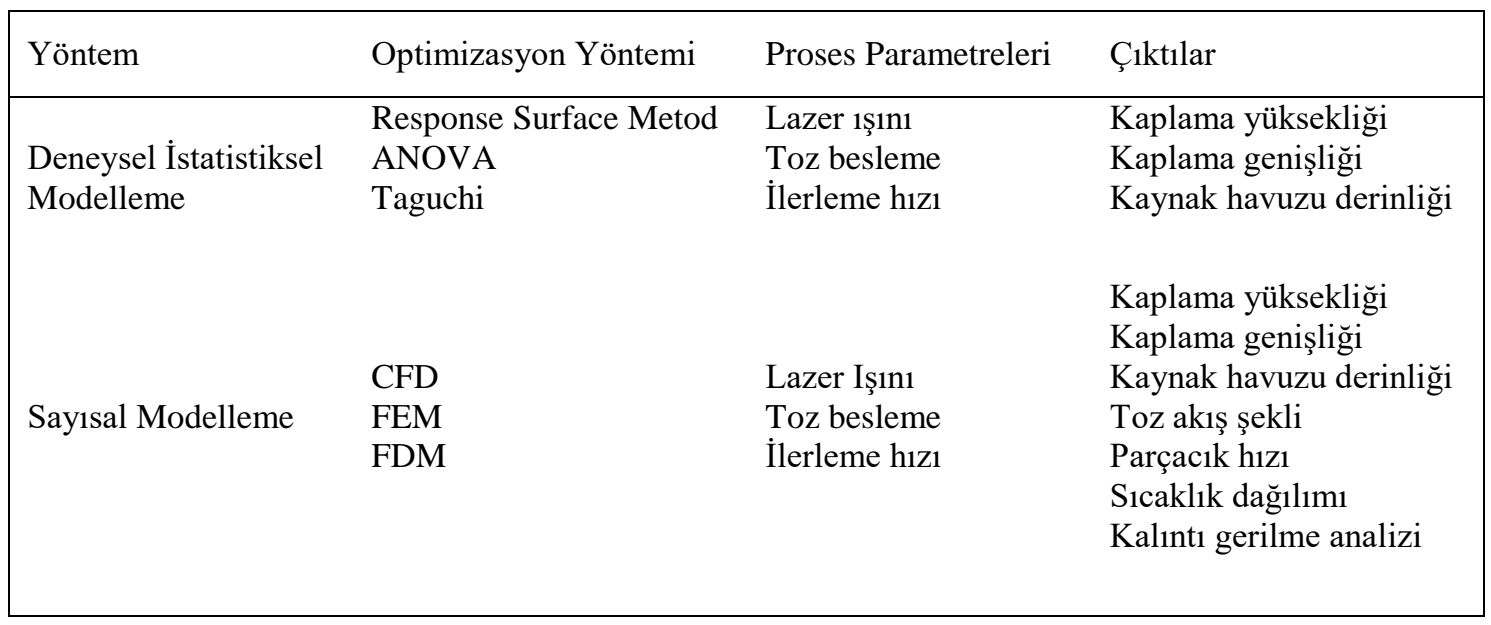

Malzeme yapısının geometrik özellikleri dolayısıyla lazer kaplama proses parametreleri optimize edilmelidir; girinti ve çıkıntıların çok olduğu doğrusal olmayan yüzeylerde yüksek kalitede kaplama elde edebilmek için proses parametrelerinin optimum kombinasyonu belirlenmelidir (Marzban ve diğ., 2014). Proses parametrelerini optimize edebilmek için birçok çalışma yapılmıştır (Calleja ve diğ., 2014; Marzban ve diğ., 2014).

Mondal ve diğ. (2013) Taguchi deney tasarımında ortagonal L9 dizisini kullanarak AISI 1040 çeliklerde farklı işlem parametreleri ile çalışmışlardır. Çalışma sonucunda, çoktan aza doğru lazer gücü, ilerleme hızı ve besleme hızlarının kaplama özellikleri kalitesi üzerinde etkili olduğunu bulmuşlardır. Marzban ve diğ. (2014) AISI 1040 çeliklerde kaplama yüksekliği, kaplama genişliği ve kaplama derinliği üzerinde proses faktörlerinin etkisini araştırmışlardır. L9 ortagonal dizisine göre deney tasarımı yapılmış ve temel bileşen analizi kullanılarak lazer kaplama işleminin çoklu yanıt optimizasyonu için TOPSIS yöntemiyle entegre edilmiştir. Sonuçlar, lazer gücünün lazer kaplama kalitesi özellikleri üzerinde en büyük etkiye sahip olduğunu göstermiştir.

Davim ve diğ. (2006) istatistiksel analiz kullanarak Ni bazlı kaplamanın geometrik formu ve sertliği üzerine deneysel bir çalışma yapmışlardır: lazer gücünün penetrasyon derinliği (\% 77.9) ve kaplama sertliği (\% 62.1) üzerinde istatistiksel ve fiziksel etki gösteren işlem parametresi olduğunu tespit etmişlerdir.

\section{SONUÇLAR}

Lazer kaplama eklemeli imalat yöntemi prototip, imalat ve hasarlı bölgenin onarımı konularında lider bir teknolojidir. Genellikle yüksek maliyete sahip kalıpların onarımında kullanılmak üzere; gövde bileşenleri, medikal implant, türbin kanadı, otomotiv çelikleri, takım çelikleri, motor krank mili, demiryolu tekerleri, dişli çarklar vb. uygulamaları da bulunmaktadır. Malzeme yüzeyinde diğer geleneksel metodlara göre üstün metalurjik özelliklere sahiptir: yorulma dayanımı, korozyon direnci, çekme dayanımı gibi mekanik özellikler istenilen düzeylerde elde edilebilmektedir.

Kaplama kalitesini etkileyen proses parametreleri etki derecesinin büyüklüğüne göre: lazer gücü, ilerleme hızı ve toz besleme hızıdır. Lazer gücündeki artış ile toz malzeme verimi ve kaplama kalınlığı artmakta; ilerleme hızındaki artış ile toz verimi düşmekte ve kaplama kalınlığ azalmaktadır. Düşük enerji girdisi gözenek oluşumuna neden olmaktadır; porozite ve çatlak 
oluşumu istenmeyen durumlardır. Malzemede istenilen mikroyapı ve mekanik özellikleri elde edebilmek için genellikle optimizasyon çalışmaları yapılmaktadır.

Lazer kaplama işlemi sırasında kaynak havuzunda hızlı katılaşma, güçlü sıvı konveksiyonu, yüzey gerilimi kaynaklı marangoni akışı, basınç değişikliği kaynaklı sıçrama etkisi ve difüzyon olayları gibi karmaşık taşınım olayları gerçekleşmektedir. Kaplama tabakasının mikroyapı özellikleri de doğrudan bu olaylar ile ilişkili olmaktadır.

Kaynak bölgesindeki sıcaklığın kontrol edilmesi önem arz etmektedir; sıcaklık alanının değişimi ile mikroyapıda tane boyutları irileşmekte ve kalıntı gerilmelere neden olarak deformasyona neden olmakta, malzemenin mekanik özelliklerini değiştirmektedir.

\section{ÇIKAR ÇATIŞMASI}

Yazarlar, bilinen herhangi bir çıkar çatışması veya herhangi bir kurum/kuruluş ya da kişi ile ortak çıkar bulunmadığını onaylamaktadırlar.

\section{YAZAR KATKISI}

Hakan AYDIN ve Çiğdem DINDAR, çalışmanın kavramsal ve tasarım süreçlerinin belirlenmesi, Çiğdem DİNDAR ve Meryem ALTAY ayrıca veri toplama aşamalarında katkı sağlamışlardır. Tüm yazarlar; çalışmanın kavramsal ve tasarım süreçlerinin yönetiminde, veri analizi ve yorumlama, makale taslağının oluşturulması ve fiziksel içeriğin incelenmesi kısımlarına katkı sağlamış olup çalışmanın son onay ve tam sorumluluğunu üstlenmektedirler.

\section{KAYNAKLAR}

1. Al-Hamdani, K. S., Murray, J. W., Hussain, T., Clare, A. T. (2020) Controlling ceramicreinforcement distribution in laser cladding of MMCs, Surface and Coatings Technology, 381, 125128. https://doi.org/10.1016/j.surfcoat.2019.125128.

2. Barr, C., Da, S., Easton, M., Orchowski, N., Matthews, N. (2018) Influence of macrosegregation on solidification cracking in laser clad ultra-high strength steels, Surface \& Coatings Technology, 340, 126-136. https://doi.org/10.1016/j.surfcoat.2018.02.052

3. Bartkowski, D., Młynarczak, A., Piasecki, A., Dudziak, B., Gos̈ciański, M., Bartkowska, A. (2015) Microstructure, microhardness and corrosion resistance of Stellite-6 coatings reinforced with WC particles using laser cladding, Optics and Laser Technology, 68, 191201. https://doi.org/10.1016/j.optlastec.2014.12.005

4. Bu, R., Jin, A., Sun, Q., Zan, W., He, R. (2020) Study on laser cladding and properties of AZ63-Er alloy for automobile engine, Journal of Materials Research and Technology, 1-7. https://doi.org/10.1016/j.jmrt.2020.03.032

5. Calleja, A., Tabernero, I., Fernández, A., Celaya, A., Lamikiz, A., López De Lacalle, L. N. (2014) Improvement of strategies and parameters for multi-axis laser cladding operations, Optics and Lasers in Engineering, 56, 113-120. https://doi.org/10.1016/j.optlaseng.2013.12.017

6. Cavaliere, P. (2021) Laser Cladding of Metals, Springer Nature. https://doi.org/10.1007/978-3-030-53195-9

7. Chen, C., Wang, Y., Ou, H., He, Y., Tang, X. (2014) A review on remanufacture of dies and moulds, Journal of Cleaner Production, 64, 13-23. https://doi.org/10.1016/j.jclepro.2013.09.014 
8. Chen, H., Lu, Y., Sun, Y., Wei, Y., Wang, X., Liu, D. (2020) Coarse TiC particles reinforced H13 steel matrix composites produced by laser cladding, Surface and Coatings Technology, 125867. https://doi.org/10.1016/j.surfcoat.2020.125867

9. Chen, J., Wang, S. H., Xue, L. (2012) On the development of microstructures and residual stresses during laser cladding and post-heat treatments, Journal of Materials Science, 47(2), 779-792. https://doi.org/10.1007/s10853-011-5854-4

10. Chen, T., Wu, W., Li, W., Liu, D. (2019) Laser cladding of nanoparticle TiC ceramic powder: Effects of process parameters on the quality characteristics of the coatings and its prediction model, Optics and Laser Technology, 116, 345-355. https://doi.org/10.1016/j.optlastec.2019.03.048

11. Chew, Y., Pang, J. H. L., Bi, G., \& Song, B. (2015). Thermo-mechanical model for simulating laser cladding induced residual stresses with single and multiple clad beads. Journal of Materials Processing Technology, 224, 89-101. https://doi.org/https://doi.org/10.1016/j.jmatprotec.2015.04.031

12. Davim, J. P., Oliveira, C., Cardoso, A. (2006) Laser cladding: An experimental study of geometric form and hardness of coating using statistical analysis, Proceedings of the Institution of Mechanical Engineers, Part B: Journal of Engineering Manufacture, 220(9), 1549-1554. https://doi.org/10.1243/09544054JEM641

13. Devojno, O. G., Feldshtein, E., Kardapolava, M. A., Lutsko, N. I. (2018) On the formation features, microstructure and microhardness of single laser tracks formed by laser cladding of a NiCrBSi self-fluxing alloy, Optics and Lasers in Engineering, 106, 32-38. https://doi.org/https://doi.org/10.1016/j.optlaseng.2018.02.004

14. El Cheikh, H., Courant, B., Branchu, S., Hascoët, J.-Y., Guillén, R. (2012) Analysis and prediction of single laser tracks geometrical characteristics in coaxial laser cladding process, Optics and Lasers in Engineering, 50(3), 413-422. https://doi.org/https://doi.org/10.1016/j.optlaseng.2011.10.014

15. Farahmand, P., Kovacevic, R. (2014) An experimental-numerical investigation of heat distribution and stress field in single- and multi-track laser cladding by a high-power direct diode laser, Optics and Laser Technology, 63, 154-168. https://doi.org/10.1016/j.optlastec.2014.04.016

16. Fu, Y., Guo, N., Cheng, Q., Zhang, D., Feng, J. (2020) In-situ formation of laser-cladded layer on Ti-6Al-4 V titanium alloy in underwater environment, Optics and Lasers in Engineering, 131(2). https://doi.org/10.1016/j.optlaseng.2020.106104

17. Gao, J., Wu, C., Hao, Y., Xu, X., Guo, L. (2020) Numerical simulation and experimental investigation on three-dimensional modelling of single-track geometry and temperature evolution by laser cladding, Optics and Laser Technology, 129, 106287. https://doi.org/10.1016/j.optlastec.2020.106287

18. Hemmati, I., Ocelík, V., De Hosson, J. T. M. (2011) The effect of cladding speed on phase constitution and properties of AISI 431 stainless steel laser deposited coatings, Surface and Coatings Technology, 205(21-22), 5235-5239. https://doi.org/10.1016/j.surfcoat.2011.05.035

19. Hofman, J. T., de Lange, D. F., Pathiraj, B., Meijer, J. (2011) FEM modeling and experimental verification for dilution control in laser cladding, Journal of Materials Processing Technology, 211(2), 187-196. https://doi.org/https://doi.org/10.1016/j.jmatprotec.2010.09.007 
20. Huang, L., Zhou, J., Xu, J., Huo, K., He, W., Meng, X., Huang, S. (2020) Microstructure and wear resistance of electromagnetic field assisted multi-layer laser clad Fe901 coating, Surface and Coatings Technology, 125876. https://doi.org/10.1016/j.surfcoat.2020.125876

21. Huang, Y., Khamesee, M. B., Toyserkani, E. (2016) A comprehensive analytical model for laser powder-fed additive manufacturing, Additive Manufacturing, 12, 90-99. https://doi.org/10.1016/j.addma.2016.07.001

22. Jiang, Y., Cheng, Y., Zhang, X., Yang, J., Yang, X. (2020) Optik Simulation and experimental investigations on the e ff ect of Marangoni convection on thermal fi eld during laser cladding process, Optik - International Journal for Light and Electron Optics, 203, 164044. https://doi.org/10.1016/j.ijleo.2019.164044

23. Kaierle, S., Overmeyer, L., Alfred, I., Rottwinkel, B., Hermsdorf, J., Wesling, V., Weidlich, N. (2017) CIRP Journal of Manufacturing Science and Technology Single-crystal turbine blade tip repair by laser cladding and remelting, CIRP Journal of Manufacturing Science and Technology, 19, 196-199. https://doi.org/10.1016/j.cirpj.2017.04.001

24. Karş1, A. (2019). Otomotivde lazer dolgu kaynağ 1 parametrelerinin etkilerinin araştırılması, Yüksek Lisans Tezi, U.Ü. Fen Bilimleri Enstitüsü, Bursa.

25. Kattire, P., Paul, S., Singh, R., Yan, W. (2015) Experimental characterization of laser cladding of CPM 9V on H13 tool steel for die repair applications, Journal of Manufacturing Processes, 20, 492-499. https://doi.org/10.1016/j.jmapro.2015.06.018

26. Li-Yan, L., Yu, Z., Yun-Jie, J., Yan, L., Hong-Fang, T., Yu-Jun, C., Cheng-Xin, L. (2020) High speed laser cladded Ti-Cu-NiCoCrAlTaY burn resistant coating and its oxidation behavior, Surface and Coatings Technology, 392, 125697. https://doi.org/10.1016/j.surfcoat.2020.125697

27. Li, C., Sun, S., Zhang, Y., Liu, C., Deng, P., Zeng, M., Wang, F., Ma, P., Li, W., Wang, Y. (2019) Effects of laser processing parameters on microstructure and mechanical properties of additively manufactured AlSi10Mg alloys reinforced by TiC, International Journal of Advanced Manufacturing Technology, 103(5-8), 3235-3246. https://doi.org/10.1007/s00170-019-04001-9

28. Li, X., Li, T., Shi, B., Wang, D., Adnan, M., Lu, H. (2020) The influence of substrate tilt angle on the morphology of laser cladding layer, Surface and Coatings Technology, 391, 125706. https://doi.org/10.1016/j.surfcoat.2020.125706

29. Liu, H., Hu, Z., Qin, X., Wang, Y., Zhang, J. (2017) Parameter optimization and experimental study of the sprocket repairing using laser cladding, Int $J$ Adv Manuf Technology, 91:3967-3975. https://doi.org/10.1007/s00170-017-0066-y

30. Liu, J., Yu, H., Chen, C., Weng, F., Dai, J. (2017) Research and development status of laser cladding on magnesium alloys: A review, Optics and Lasers in Engineering, 93, 195-210. https://doi.org/10.1016/j.optlaseng.2017.02.007

31. Locs, S., Boiko, I., Leitans, A., Drozdovs, P. (2017) Experimental study of coaxial laser cladding of tool steel, Engineering for Rural Development, 16, 1038-1046. https://doi.org/10.22616/ERDev2017.16.N219

32. Lourenço, J. M., Sun, S. Da, Sharp, K., Luzin, V., Klein, A. N., Wang, C. H., Brandt, M. (2016) Fatigue and fracture behavior of laser clad repair of AerMet ${ }^{\circledR} 100$ ultra-high strength steel, International Journal of Fatigue, 85, 18-30. https://doi.org/10.1016/j.ijfatigue.2015.11.021 
33. Lu, J. Z., Cao, J., Lu, H. F., Zhang, L. Y., Luo, K. Y. (2019) Wear properties and microstructural analyses of Fe-based coatings with various WC contents on $\mathrm{H} 13$ die steel by laser cladding, Surface \& Coatings Technology, 369, 228-237. https://doi.org/10.1016/j.surfcoat.2019.04.063

34. Ma, M., Xiong, W., Lian, Y., Han, D., Zhao, C., Zhang, J. (2020) Modeling and optimization for laser cladding via multi-objective quantum-behaved particle swarm optimization algorithm, Surface and Coatings Technology, 381, 125129. https://doi.org/10.1016/j.surfcoat.2019.125129

35. Marin, E., Zanocco, M., Boschetto, F., Santini, M., Zhu, W., Adachi, T., Ohgitani, E., McEntire, B. J., Bal, B. S., Pezzotti, G. (2020) Silicon nitride laser cladding: A feasible technique to improve the biological response of zirconia, Materials \& Design, 191, 108649. https://doi.org/10.1016/j.matdes.2020.108649

36. Marques, M. J., Ramasamy, A., Batista, A. C., Nobre, J. P., Loureiro, A. (2015) Effect of heat treatment on microstructure and residual stress fields of a weld multilayer austenitic steel clad, Journal of Materials Processing Technology, 222, 52-60. https://doi.org/10.1016/j.jmatprotec.2015.03.004

37. Marzban, J., Ghaseminejad, P., Ahmadzadeh, M. H., Teimouri, R. (2014) Experimental investigation and statistical optimization of laser surface cladding parameters, International Journal of Advanced Manufacturing Technology, 76(5-8), 1163-1172. https://doi.org/10.1007/s00170-014-6338-x

38. Mondal, S., Paul, C. P., Kukreja, L. M., Bandyopadhyay, A., Pal, P. K. (2013) Application of Taguchi-based gray relational analysis for evaluating the optimal laser cladding parameters for AISI1040 steel plane surface, International Journal of Advanced Manufacturing Technology, 66(1-4), 91-96. https://doi.org/10.1007/s00170-012-4308-8

39. Moskal, G., Niemiec, D., Chmiela, B., Kałamarz, P., Durejko, T., Ziętala, M., Czujko, T. (2020) Microstructural characterization of laser-cladded NiCrAlY coatings on Inconel 625 Ni-based superalloy and 316L stainless steel, Surface and Coatings Technology, 387, 125317. https://doi.org/10.1016/j.surfcoat.2019.125317

40. Muvvala, G., Patra Karmakar, D., Nath, A. K. (2017) Online monitoring of thermo-cycles and its correlation with microstructure in laser cladding of nickel based super alloy, Optics and Lasers in Engineering, 88, 139-152. https://doi.org/https://doi.org/10.1016/j.optlaseng.2016.08.005

41. Quazi, M. M., Fazal, M. A., Haseeb, A. S. M. A., Yusof, F., Masjuki, H. H., Arslan, A. (2016) Effect of rare earth elements and their oxides on tribo-mechanical performance of laser claddings: A review, Journal of Rare Earths, 34(6), 549-564. https://doi.org/10.1016/S1002-0721(16)60061-3

42. Reddy, L., Preston, S. P., Shipway, P. H., Davis, C., Hussain, T. (2018) Process parameter optimisation of laser clad iron based alloy: Predictive models of deposition efficiency, porosity and dilution, Surface and Coatings Technology, 349, 198-207. https://doi.org/https://doi.org/10.1016/j.surfcoat.2018.05.054

43. Ren, H., Ren, J., Zhao, L., Wang, Q. (2013) Simulation Analysis on Selection of Laser Cladding Repair Material for the Diesel Engine Crankshaft Crack, Advanced Materials Research, 820, 175-179. https://doi.org/10.4028/www.scientific.net/AMR.820.175

44. Riveiro, A., Mejías, A., Lusquiños, F., del Val, J., Comesaña, R., Pardo, J., Pou, J. (2014) Laser cladding of aluminium on AISI 304 stainless steel with high-power diode lasers, 
Dindar Ç., Altay M.,Aydın H.: Lazer Kaplama Prosesi ve Proses Parametreleri Derleme Çalışması

Surface and Coatings Technology, 253, 214-220. https://doi.org/https://doi.org/10.1016/j.surfcoat.2014.05.039

45. Roy, T., Paradowska, A., Abrahams, R., Law, M., Mutton, P., Soodi, M., Yan, W. (2020) Residual stress in laser cladded heavy-haul rails investigated by neutron diffraction, Journal of Materials Processing Technology, 278, 116511. https://doi.org/10.1016/j.jmatprotec.2019.116511

46. Singh, S., Ramakrishna, S., Singh. R. (2017) Material issues in additive manufacturing A review, Journal of Manufacturing Processes, 25, 185-200. doi:10.1016/j.jmapro.2016.11.006

47. Shamsaei, N., Yadollahi, A., Bian, L., Thompson, S. M. (2015) An overview of Direct Laser Deposition for additive manufacturing; Part II: Mechanical behavior, process parameter optimization and control, Additive Manufacturing, 8, 12-35. https://doi.org/10.1016/j.addma.2015.07.002

48. Shi, J., Zhu, P., Fu, G., Shi, S. (2018) Geometry characteristics modeling and process optimization in coaxial laser inside wire cladding, Optics \& Laser Technology, 101, 341348. https://doi.org/https://doi.org/10.1016/j.optlastec.2017.10.035

49. Shi, Y., Jin, H., Wu, P. D., Lloyd, D. J. (2017) Acta Materialia Analysis of roping in an AA6111 T4P automotive sheet in 3D deformation states, Acta Materialia, 124, 598-607. https://doi.org/10.1016/j.actamat.2016.11.028

50. Şimşek, T., İzciler, M., Ozcan, Ş., Akkurt, A. (2019) Laser Cladding of Hot Work Tool Steel (H13) With Nano Tic Particles, Turkish Journal of Engineering, 3(1), 1-10. https://doi.org/10.31127/tuje.419531

51. Siva Prasad, H., Brueckner, F., Volpp, J., Kaplan, A. F. H. (2020) Laser metal deposition of copper on diverse metals using green laser sources, International Journal of Advanced Manufacturing Technology, 107(3-4), 1559-1568. https://doi.org/10.1007/s00170-02005117-z

52. Sun, G., Tong, Z., Fang, X., Liu, X., Ni, Z., Zhang, W. (2016) Effect of scanning speeds on microstructure and wear behavior of laser-processed $\mathrm{NiCr}-\mathrm{Cr} 3 \mathrm{C} 2-\mathrm{MoS} 2-\mathrm{CeO} 2$ on 38CrMoAl steel, Optics \& Laser Technology, 77, 80-90. https://doi.org/https://doi.org/10.1016/j.optlastec.2015.08.008

53. Tabernero, I., Lamikiz, A., Ukar, E., Arregi, B., Figueras, J., Soriano, C. (2009) Parameter optimization for mould and die recovering using laser cladding, AIP Conference Proceedings, 1181, 484-493. https://doi.org/10.1063/1.3273666

54. Tamanna, N., Crouch, R., Naher, S. (2019) Progress in numerical simulation of the laser cladding process, Optics and Lasers in Engineering, 122, 151-163. https://doi.org/10.1016/j.optlaseng.2019.05.026

55. Theron, M., Rooyen, C. Van, Malabi, K. (2020) Investigation into laser refurbishment and transformation hardening of cast iron forming dies for the automotive industry Investigation into laser refurbishment and transformation hardening of cast iron forming dies for the automotive industry, 022071. https://doi.org/10.2351/7.0000106

56. Toyserkani, E., Khajepour, A., Corbin, S. (2004) Laser Cladding, CRC Press, New York. https://doi.org/10.1201/9781420039177

57. Wang, Q., Chen, F. Q., Zhang, L., Li, J. D., Zhang, J. W. (2020) Microstructure evolution and high temperature corrosion behavior of $\mathrm{FeCrBSi}$ coatings prepared by laser cladding, Ceramics International, 46, 17233-17242. https://doi.org/10.1016/j.ceramint.2020.04.010 
58. Weng, F., Chen, C., Yu, H. (2014) Research status of laser cladding on titanium and its alloys: A review, Journal of Materials\&Design, 58, 412-425. https://doi.org/10.1016/j.matdes.2014.01.077

59. Xu, X., Mi, G., Xiong, L., Jiang, P., Shao, X., Wang, C. (2018) Morphologies, microstructures and properties of TiC particle reinforced Inconel 625 coatings obtained by laser cladding with wire, Journal of Alloys and Compounds, 740, 16-27. https://doi.org/10.1016/j.jallcom.2017.12.298

60. Yu, J., Rombouts, M., Maes, G. (2013) Cracking behavior and mechanical properties of austenitic stainless steel parts produced by laser metal deposition, Materials and Design, 45, 228-235. https://doi.org/10.1016/j.matdes.2012.08.078

61. Yu, T., Zhao, Y., Sun, J., Chen, Y., Qu, W. (2018) Process parameters optimization and mechanical properties of forming parts by direct laser fabrication of YCF101 alloy, Journal of Materials Processing Technology, 262, 75-84. https://doi.org/10.1016/j.jmatprotec.2018.06.023

62. Zareh, P., Urbanic, R. J. (2020) Numerical simulations for laser clad beads with a variable side-to-side overlap condition, The International Journal of Advanced Manufacturing Technology, 1027-1058. https://doi.org/10.1007/s00170-020-05565-7

63. Zhan, X., Qi, C., Gao, Z., Tian, D., Wang, Z. (2019) The influence of heat input on microstructure and porosity during laser cladding of Invar alloy, Optics and Laser Technology, 113, 453-461. https://doi.org/10.1016/j.optlastec.2019.01.015

64. Zhang, Z., Farahmand, P., Kovacevic, R. (2016) Laser cladding of 420 stainless steel with molybdenum on mild steel A36 by a high power direct diode laser, Materials \& Design, 109, 686-699. https://doi.org/https://doi.org/10.1016/j.matdes.2016.07.114

65. Zhao, J., Wang, G., Wang, X., Luo, S., Wang, L., Rong, Y. (2020a) Multicomponent multiphase modeling of dissimilar laser cladding process with high-speed steel on medium carbon steel, International Journal of Heat and Mass Transfer, 148, 118990. https://doi.org/10.1016/j.ijheatmasstransfer.2019.118990

66. Zhao, Y., Yu, T., Sun, J., Jiang, S. (2020b) Microstructure and properties of laser cladded B4C/TiC/Ni-based composite coating, International Journal of Refractory Metals and Hard Materials, 86, 105112. https://doi.org/10.1016/j.ijrmhm.2019.105112

67. Zheng, M., Fan, D., Li, X. K., Li, W. F., Liu, Q. B., Zhang, J. B. (2008) Microstructure and osteoblast response of gradient bioceramic coating on titanium alloy fabricated by laser cladding, Applied Surface Science, 255(2), 426-428. https://doi.org/10.1016/j.apsusc.2008.06.078

68. Zhu, L., Wang, S., Pan, H., Yuan, C., Chen, X. (2020) Research on remanufacturing strategy for 45 steel gear using H13 steel powder based on laser cladding technology, Journal of Manufacturing Processes, 49, 344-354. https://doi.org/10.1016/j.jmapro.2019.12.009

69. Zhu, Y., Yang, Y., Mu, X., Wang, W., Yao, Z., Yang, H. (2019) Study on wear and RCF performance of repaired damage railway wheels : Assessing laser cladding to repair local defects on wheels, Wear, 431, 126-136. https://doi.org/10.1016/j.wear.2019.04.028 
